\title{
DURAÇÃO DO PERÍODO DE MOLHAMENTO FOLIAR EM POMARES DE MACIEIRA EM CÉU ABERTO E SOB TELA ANTIGRANIZO, EM VACARIA-RS ${ }^{1}$
}

\author{
VIVIANE AIRES DE PAULA ${ }^{2}$, HOMERO BERGAMASCHI ${ }^{3}$, EMERSON MEDEIROS DEL PONTE ${ }^{4}$, \\ LOANA SILVEIRA CARDOSO ${ }^{5}$, LEOSANE CRISTINA BOSCO ${ }^{6}$
}

RESUMO - A duração do período de molhamento foliar (DPM) é um importante parâmetro para a determinação do risco de doenças da macieira, especialmente a sarna, principal doença da cultura. O clima regional e as características de implantação e manejo de pomares são fatores determinantes da ocorrência do molhamento foliar. Em função do microclima do dossel, é necessário conhecer a posição com maior DPM, para dar suporte a modelos de previsão de doenças. Poucos trabalhos avaliaram a DPM em pomares de macieira, sendo que inexistem estudos nas condições de cultivo do Sul do Brasil, em particular na região produtora de Vacaria, Rio Grande do Sul. Este trabalho objetivou caracterizar a variação espacial da DPM em dosséis de macieiras em céu aberto e sob tela antigranizo, na região de Vacaria. O experimento foi desenvolvido em pomares de macieiras 'Royal Gala', conduzidos em líder central com apoio (espaçamento de $1 \mathrm{~m} \times 3 \mathrm{~m}$ ), sendo um coberto por tela antigranizo e o outro em céu aberto. A DPM foi monitorada nos estratos superior, médio e inferior dos dosséis de macieira. A duração do molhamento foliar foi superior no estrato inferior do dossel, tanto em céu aberto quanto sob tela antigranizo. Houve tendência de mais alta DPM no pomar coberto para todos os estratos do dossel, com diferença significativa entre ambientes nos estratos superior e médio. Os valores de DPM foram mais similares entre os estratos no dossel coberto por tela antigranizo do que no dossel a céu aberto.

Termos para indexação: Malus domestica, doenças, microclima, orvalho.

\section{LEAF WETNESS DURATION IN APPLE ORCHARDS IN OPEN SKY AND UNDER HAIL NET COVER, IN VACARIA, BRAZIL}

\begin{abstract}
The leaf wetness duration (LWD) is an important parameter to monitor the epidemiology of apple diseases, like apple scab, the most common disease of the crop. The regional climate and management conditions of the orchards are determinant factors for the occurrence of leaf wetness. And, depending on the canopy microclimate it is important to know the position with greater LWD, for use in forecasting models of disease. Few studies have evaluated LWD on apple orchards and none in the cropping conditions of southern Brazil, particularly in the producing region of Vacaria, in the state of Rio Grande do Sul, Brazil. This study aimed to characterize the spatial variation of LWD in canopies of apple trees in open sky and under hail net covering, in Vacaria, Brazil. The experiment was carried out in orchards of 'Royal Gala' apple cultivars, conducted in central leader with support (spacing $1 \mathrm{~m} \times 3 \mathrm{~m}$ ), being one in open sky and other under hail net. The LWD was monitored in the upper, middle and lower strata of the plant canopy. The LWD was higher in the lower part of the canopy in both apple orchards. Tendency to greater LWD in the covered orchard was observed for all strata of the canopy, with significant differences for the upper and middle strata of canopies. LWD values were more similar between portions of the canopy under hail net than in the open sky.
\end{abstract}

Index terms: Malus domestica, wetness, diseases, microclimate.

\footnotetext{
'(Trabalho 219-11). Recebido em:29-08-2011. Aceito para publicação em: 10-04-2012.

${ }^{2}$ Eng. Agrônoma, Doutora em Fitotecnia pelo PPG Fitotecnia, Faculdade de Agronomia, UFRGS, Av. Bento Gonçalves, 7712, CEP: 91540-000, Porto Alegre-RS. E-mail: vivianeadp@yahoo.com.br - Bolsista CNPq

${ }^{3}$ Eng. Agrônomo, Prof. Doutor, Dep. Plantas Forrageiras e Agrometeorologia, Faculdade de Agronomia, UFRGS, Av. Bento Gonçalves, 7712, CEP: 91540-000, Porto Alegre-RS. E-mail: homerobe@ufrgs.br - Bolsista CNPq

${ }^{4}$ Eng. Agrônomo, Prof. Doutor, Dep. Fitossanidade, Faculdade de Agronomia, UFRGS, Av. Bento Gonçalves, 7712, CEP: 91540-000, Porto Alegre-RS. E-mail: emerson.delponte@ufrgs.br

${ }_{5}^{5}$ Eng. Agrônoma, Doutora em Fitotecnia pelo PPG Fitotecnia, Faculdade de Agronomia, UFRGS, Av. Bento Gonçalves, 7712, CEP: 91540-000, Porto Alegre-RS. E-mail: loanacar@yahoo.com.br - Bolsista CNPq

${ }^{6}$ Eng. Agrônoma, Doutora do PPG Fitotecnia, Faculdade de Agronomia, UFRGS, Av. Bento Gonçalves, 7712, CEP: 91540-000, Porto Alegre-RS. E-mail: leosaneb@yahoo.com.br - Bolsista CNPq
} 


\section{INTRODUÇÃO}

O molhamento foliar é um termo genérico utilizado para designar a presença de água na forma líquida sobre a parte aérea dos vegetais, podendo ser provocado por orvalho, neblina, chuva ou irrigação (HUBER; GILLESPIE, 1992). A duração do período de molhamento foliar (DPM) é um importante fator epidemiológico de doenças de plantas, uma vez que desempenha papel fundamental em alguns processos do ciclo, tais como a infecção e a esporulação (VALE et al., 2004).

A DPM e a temperatura do ar são variáveis amplamente utilizadas como entrada em modelos usados em sistemas de alerta de risco de doenças (HUBER; GILLESPIE, 1992). A exemplo, elas são utilizadas em modelos e sistemas de alerta de algumas doenças da macieira, como alternaria (Alternaria mali), podridão-amarga (Glomerella cingulata) e sarna-da-macieira (Venturia inaequalis) (BERTON, 2004)

A cultura da macieira tem grande importância econômica no Sul do Brasil. No entanto, a ocorrência de doenças é fator limitante, sendo a sarna-da-macieira a principal delas. Sistemas de alerta fitossanitário e modelos de simulação para sarna-da-macieira foram desenvolvidos com base na Tabela de Mills, que inter-relaciona DPM e temperatura do ar com a intensidade da doença (MILLS, 1944; MacHARDY; GADOURY, 1989; E MacHARDY,1996).

A temperatura do ar pode ser mais facilmente monitorada, mas a DPM é considerada de difícil medição ou estimativa, em razão de não estar relacionada apenas às variáveis atmosféricas, mas também às propriedades ópticas da superfície e ao microclima (MADEIRA et al., 2002).

A variabilidade espacial da duração do molhamento foliar é um aspecto importante a ser considerado nas medições em cultivos agrícolas (SENTELHAS et al., 2005). O microclima de uma cultura é influenciado por fatores como área foliar, estrutura das plantas, sistema de cultivo, distribuição das plantas no terreno, altura das plantas, entre outros (MONTEITH, 1990; MADEIRA et al., 2002). Estes fatores controlam a interceptação e o balanço de radiação, determinando os regimes de temperatura, umidade do ar e vento dentro dos cultivos que, por sua vez, irão influenciar na duração do molhamento, permitindo que várias partes das plantas se tornem úmidas ou secas em diferentes momentos (HUBER; GILLESPIE, 1992).

O microclima dos cultivos também pode ser influenciado pelo uso de coberturas, a exemplo das coberturas plásticas e das telas antigranizo. Estes tipos de cobertura vêm sendo cada vez mais utilizados, e suas influências no microclima vêm sendo e devem ser estudadas, inclusive em frutíferas de clima temperado, como telas antigranizo em macieiras (LEITE et al., 2002; AMARANTE et al., 2009; CARDOSO et al., 2009; e BOSCO et al., 2010) e coberturas plásticas em vinhedos (CARDOSO et al., 2010; CHAVARRIA et al., 2007; CHAVARRIA et al., 2008; CHAVARRIA et al., 2009). Quanto ao efeito sobre o microclima, da utilização de cobertura plástica para diferentes culturas, os estudos estão sendo difundidos. Em frutíferas de clima temperado, foram desenvolvidos trabalhos em videiras, os quais avaliaram detalhadamente a influência do uso dessas coberturas em vinhedos na Serra Gaúcha. Contudo, no que se refere à utilização de tela antigranizo, os estudos são menos difundidos. Porém, este tipo de cobertura está sendo cada vez mais utilizado nas regiões produtoras de maçã no Brasil, inclusive na região de Vacaria, onde foram iniciados trabalhos por Cardoso et al. (2009) e Bosco et al. (2010).

A variabilidade espacial da DPM pode influenciar na determinação do risco da doença por modelos de previsão com base nessa variável (DALLA MARTA et al., 2004), de forma que seu estudo é necessário para aprimorar as medições e as estimativas da DPM em cultivos agrícolas. A partir da medição da DPM, é possível identificar as posições da cultura onde esta é mais prolongada e, consequentemente, mais propensa à ocorrência de doenças. Desta forma, as medidas ou estimativas da DPM não devem ser subestimadas sob pena de ignorarem condições críticas favoráveis à ocorrência de uma doença. Também, devem-se evitar superestimativas da DPM quando elas forem utilizadas em tomadas de decisão no manejo das doenças. Muito embora seja de grande importância, a variabilidade da DPM em diferentes culturas não tem sido amplamente estudada.

$\mathrm{Na}$ cultura da macieira, existem poucos trabalhos tanto para avaliação espacial como para estimativa da DPM, sendo que nenhum deles ocorreu nas condições de cultivo predominantes no Rio Grande do Sul, assim como com uso de cobertura por tela antigranizo. Esses foram desenvolvidos em locais com condições climáticas específicas, como os desenvolvidos por Batzer et al. (2008) e Sentelhas et al. (2005), ambos nos Estados Unidos. Além disso, aspectos relacionados à cultivar, densidade de plantas e sistema de condução da cultura tendem a ser diferentes dos que predominam nas principais regiões de cultivo do Sul do Brasil.

Diante do exposto, o objetivo deste trabalho foi caracterizar a variação espacial da duração do molha- 
mento foliar em dosséis de macieiras a céu aberto ou sob tela antigranizo, a fim de caracterizar e comparar a DPM nas duas condições, bem como melhorar o desempenho de modelos de estimativa desta variável, na região de Vacaria, Rio Grande do Sul.

\section{MATERIAL E MÉTODOS}

O estudo foi desenvolvido em pomar comercial da Empresa Schio Agropecuária Ltda., no Município de Vacaria-RS, de outubro de 2009 a março de 2010. Utilizou-se a cultivar de macieira 'Royal Gala', sobre porta-enxerto $\mathrm{M} 9$, com 10 anos de implantação e espaçamentos de $1,0 \mathrm{~m}$ x 3,5 m. As fileiras tinham direção norte-sul, com plantas conduzidas em líder central com apoio.

Foram utilizadas duas quadras de pomar distanciadas em $15 \mathrm{~m}$, sendo uma conduzida em céu aberto (descoberto) e a outra sob cobertura de tela antigranizo (coberto). Foi utilizada tela antigranizo de cor preta, com malhas de $4 \times 7 \mathrm{~mm}$, instalada um ano após o plantio das mudas. Esta foi apoiada sobre estrutura fixa, formando duas águas com abertura de 20 a $30 \mathrm{~cm}$ nas entrelinhas, para escoamento do granizo. A distância entre o topo das plantas e a tela era de aproximadamente $30 \mathrm{~cm}$. Com amostras aleatórias de 10 plantas, foram determinados: altura média de plantas de 2,7 m em céu aberto e 3,0 m no pomar coberto; diâmetro médio na base do tronco de $4,5 \mathrm{~cm}$ nos dois ambientes; e média de 17 ramos por planta nos dois ambientes.

Com a utilização de porta-enxerto de baixo vigor, sistema de condução e espaçamento em alta densidade, configurou-se um dossel com plantas pouco vigorosas, formando linhas espaçadas e abertas. Além disso, as plantas foram submetidas a podas verdes de desbaste, durante o período de pleno crescimento vegetativo.

No interior de cada quadra (ambientes com e sem cobertura de tela antigranizo) e numa linha de plantas, foram instalados sensores para monitorar a duração do período de molhamento (DPM). Para tanto, foram utilizados dois tipos de sensores: o modelo 237-L (Campbell) e sensores com placa de PVC desenvolvidos pela Epagri (Empresa de Pesquisa Agropecuária e Extensão Rural de Santa Catarina), aqui denominados Placas de Molhamento Foliar da Epagri (PMFE). Todos os sensores de molhamento foliar foram instalados em pares, para cada posição do dossel em que a DPM foi medida.

A DPM foi medida nos extratos superior, médio e inferior dos pomares descoberto (em céu aberto) e coberto (abaixo da tela). Os sensores foram instalados com inclinação de $45^{\circ} \mathrm{e}$ voltados para o sul, segundo recomendações de Sentelhas (2004). A DPM foi totalizada para períodos de 24 horas, iniciando-se às $12 \mathrm{~h} 30 \mathrm{~min}$ do dia " $\mathrm{n}$ ", encerrando-se às $12 \mathrm{~h}$ do dia " $\mathrm{n}+1$ ".

Todos os sensores foram conectados a um sistema automático de aquisição de dados, composto por um multiplexador AM 416 de 40 canais, um datalogger CR21X e uma unidade armazenadora acoplada (todos da marca Campbell), com leituras realizadas a cada 30 s e registros a cada meia hora.

Antes da utilização no campo, os sensores de molhamento (237-L e PMFE) foram pintados com tinta látex de cor branca, visando a aumentar a sensibilidade do sensor ao molhamento e simular às propriedades ópticas das folhas, de acordo com recomendação de Sentelhas (2004). Após a pintura, os sensores foram submetidos a tratamento térmico em estufa $\left(65^{\circ} \mathrm{C}\right.$ por $\left.12 \mathrm{~h}\right)$, conforme sugerido pelo mesmo autor. Após esse processo, os sensores foram testados e calibrados, de modo a estabelecer os limiares entre condições de molhado e completamente seco e para ajuste das medições entre sensores, a fim de obter valores iguais, quando instalados em condições idênticas.

Foi comparada a DPM (horas por dia) entre posições no dossel, nos terços superior, médio e inferior de cada ambiente (coberto e descoberto) e entre ambientes para mesma posição na planta. A variabilidade de DPM dentro e entre ambientes foi avaliada pelo desvio-padrão (DP), coeficiente de variação $(\mathrm{CV} \%)$, diferença absoluta média (DAM), diferença média (DM) e teste t para dados pareados, ao nível de $5 \%$ de probabilidade de erro.

Os dados de DPM dentro e entre ambientes foram avaliados pelas seguintes estatísticas: coeficiente de correlação (r) e índice de concordância de Willmot (D, Equação 1).

$D=1-\left\{\frac{\sum\left(\mathrm{O}_{i}-\mathrm{P}_{\mathrm{i}}\right)^{2}}{\sum\left(\left|\mathrm{P}_{\mathrm{i}}-\mathrm{O}_{\mathrm{m}}\right|+\mid \mathrm{O}_{\mathrm{i}}-\mathrm{O}_{\mathrm{m}}\right)^{2}}\right\}$

sendo D o coeficiente de concordância de Willmont; $\mathrm{O}_{\mathrm{i}}$ cada valor observado colocado como "referência"; $\mathrm{P}_{\mathrm{i}}$ cada valor observado a ser comparado com a "referência"; $\mathrm{O}_{\mathrm{m}}$ a média dos valores observados de "referência".

Foi realizado monitoramento em nível microclimático para temperatura e umidade do ar, radiação solar e vento, nos pomares deste estudo (coberto por tela antigranizo e em céu aberto), cujos critérios e resultados constam em Cardoso et al. (2009) e Bosco et al. (2010). 


\section{RESULTADOS E DISCUSSÃO}

No pomar em céu aberto (descoberto), a duração do molhamento foliar (DPM) diminuiu do terço inferior para o meio do dossel, e deste para o terço superior da cultura (Figura 1, Tabela 1). No pomar coberto por tela antigranizo, o padrão foi similar, mas as diferenças de DPM entre estratos do dossel foram menores que no pomar em céu aberto (Figura 1). Portanto, a DPM média do período avaliado decresceu na seqüência dos estratos inferior, médio e superior do dossel, em ambos os pomares: coberto e descoberto.

No pomar descoberto, a DPM média foi significativamente diferente entre todos os estratos (Tabela 1). Nesse pomar, as médias de DPM foram de $13,5 \mathrm{~h}$ no estrato inferior, $12,7 \mathrm{~h}$ no estrato médio e $12,3 \mathrm{~h}$ no estrato superior. As médias de DPM no pomar coberto não apresentaram diferença significativa na comparação entre os terços médio e inferior do dossel (Tabela 1). Sob tela antigranizo, as médias de DPM foram de 13,9h e 13,8h nos estratos inferior e médio e $13,2 \mathrm{~h}$ no terço superior do dossel de plantas.

A tendência de variação na duração do molhamento foliar, principalmente no pomar descoberto, foi contrária daquela encontrada em trabalho desenvolvido por Sentelhas (2004) e Sentelhas et al. (2005) em Ames, Estados Unidos, onde a DPM foi maior na sequência dos estratos superior, médio e inferior. No referido trabalho, os autores encontraram cerca de $2 \mathrm{~h}$ a mais de DPM no estrato superior de macieiras, similar ao observado por Wittich (1995). Batzer et al. (2008) também encontraram maior DPM no estrato superior do dossel de macieiras, em Iowa, nos Estados Unidos. As diferenças entre os resultados deste trabalho e aqueles obtidos pelos autores citados podem ser atribuídas, sobretudo, a diferenças na estrutura dos dosséis e, consequentemente, nas condições microclimáticas dos pomares.

Nos estudos citados, a estrutura dos dosséis e a disposição de plantas eram diferentes daquelas utilizadas no presente trabalho. Nos trabalhos desenvolvidos nos Estados Unidos por Sentelhas et al. (2005) e Batzer et al. (2008), utilizaram-se pomares de macieira 'Golden Delicious' com espaçamento de $3 \mathrm{~m}$ por $5 \mathrm{~m}$. Esses dosséis não usam porta-enxerto "ananizante" e em baixa densidade, formavam plantas robustas e copas fechadas, com altura entre 3,5 e $5 \mathrm{~m}$ e aproximadamente $2 \mathrm{~m}$ de largura. Desta forma, somente o topo das plantas ficava diretamente exposto ao céu. Assim, o estrato superior das plantas estaria mais propenso a apresen- tar molhamento, tanto em dias somente com orvalho como em dias de chuva.

No presente trabalho, as plantas de macieira 'Royal Gala' eram de porte menor, devido ao portaenxerto de baixo vigor vegetativo (M9) e aos espaçamentos de $1 \mathrm{~m}$ entre plantas e 3,5 m entre linhas, com altura de aproximadamente $3 \mathrm{~m}$ e, no máximo, $1 \mathrm{~m}$ de largura de plantas. Desta forma, os pomares configuravam-se em linhas espaçadas e abertas, à semelhança de vinhedos em espaldeira, embora com maior densidade de plantas nas linhas. Nesta configuração, a radiação solar penetra no interior do dossel, pelas entrelinhas, e as folhas ficam expostas à formação do orvalho, mesmo em estratos inferiores. Contudo, a secagem dá-se antes no estrato superior, seguindo em direção ao estrato inferior do dossel. Este aspecto pode ser explicado pelas trocas de energia radiante e pela ação do vento que tendem a ser mais intensas no topo que na base do dossel. Os dosséis em linhas espaçadas e abertas do presente trabalho configuram-se pela alta densidada e pela utilização de porta-enxerto de baixo vigoroso. Contudo, é importante ressaltar que eram feitas podas verdes de desbaste de ramos, de modo a manter esta arquitetura ao dossel. O porta-enxerto M9 é muito utilizado em pomares de alta densidade em Vacaria e, segundo Pedrotti e Voltolini (2001), também é o mais utilizado no Estado de Santa Catarina, em pomares de alta densidade, visando ao porte reduzido das plantas, o que facilita o manejo dos pomares.

Pelas características físicas e microclimáticas dos dosséis, as variações na duração do molhamento foliar, observadas no presente trabalho, assemelhamse àquelas encontradas por Sentelhas (2004) em dosséis jovens (e abertos) de cafeeiros, onde DPM foi maior na parte inferior do dossel que no topo das plantas. Por outro lado, em videiras cultivadas em espaldeira, o mesmo autor não encontrou diferenças em DPM entre as posições no dossel (SENTELHAS, 2004). Convém ressaltar que as videiras em espaldeiras apresentam largura de plantas ainda menores que os cafeeiros e as macieiras, com exposição às condições de ambiente, praticamente idêntica para todo o perfil do dossel.

No pomar coberto, a DPM foi, em média, maior que no pomar em céu aberto, para todos os estratos no dossel. Nos terços superior e médio, a DPM diferiu significativamente entre ambientes. No entanto, no terço inferior do dossel, não houve diferenças significativas entre ambientes (Tabela 1).

No pomar descoberto, a duração do molhamento foliar no terço inferior do dossel foi, em média, 
0,8 e 1,2 h maior que nos estratos médio e superior, respectivamente. Neste ambiente, DPM no estrato médio foi, em média, $0,4 \mathrm{~h}$ maior que na porção superior do dossel. Corroborando estas diferenças entre porções do dossel de macieiras, a DPM no terço inferior foi maior que no estrato intermediário em $66 \%$ dos dias, e maior que no terço superior em $73 \%$ dos dias, enquanto DPM no terço intermediário foi maior que no estrato superior em $61 \%$ dos dias (Tabela 2).

Considerando somente os dias sem chuva, as diferenças médias são iguais entre os estratos inferior e médio e entre inferior e superior. No entanto, a porção intermediária passou a ter, em média, $0,5 \mathrm{~h}$ a mais de molhamento foliar que o estrato superior. Em dias de chuva, as diferenças médias entre estratos demonstram que a DPM no terço inferior foi $0,9 \mathrm{~h}$ maior que no terço médio, no estrato inferior foi $1,1 \mathrm{~h}$ maior que no superior, e no estrato médio a DPM foi apenas $0,2 \mathrm{~h}$ maior que no terço superior (Tabela 2).

No pomar coberto por tela antigranizo, as diferenças médias de DPM entre estratos do dossel foram menores. No terço inferior, ela foi 0,2 e $0,7 \mathrm{~h}$ maior que nos estratos intermediário e superior, respectivamente. O estrato médio do dossel teve DPM $0,7 \mathrm{~h}$ maior que o terço superior. Neste pomar, a DPM na porção inferior do dossel foi superior àquela da porção intermediária em apenas $44 \%$ dos dias e igual em $33 \%$ dos dias. O terço inferior do dossel teve DPM maior que o estrato superior em $66 \%$ dos dias, e o estrato médio teve DPM maior que o estrato superior em $68 \%$ dos dias (Tabela 2).

As medições da duração do molhamento foliar podem ser consideradas precisas e acuradas, pelo elevado índice de concordância de Willmot (D), e altamente correlacionadas (r) entre diferentes estratos do dossel de cada ambiente, e entre ambientes para um mesmo estrato do dossel (Tabela 3). Os índices estatísticos foram mais elevados no pomar coberto por tela, que também apresentou menor coeficiente de variação para duração de molhamento foliar entre estratos do dossel (Figura 2).
Pela Figura 2, verifica-se que os coeficientes de variação entre valores de DPM para diferentes estratos do dossel descoberto foi superior àqueles do dossel coberto por tela, com ou sem ocorrência de precipitação pluvial. Ainda, verifica-se que, em dias de chuva, houve uma pequena redução na variação da DPM entre estratos do dossel de macieiras (Figura 2).

Segundo resultados encontrados por Cardoso et al. (2009) e Bosco et al. (2010), seguindo uma sequência de estudos no mesmo experimento, não houve diferenças entre médias diárias de temperatura e umidade relativa do ar entre os pomares em céu aberto e sob tela antigranizo, num mesmo estrato do dossel. No entanto, os autores verificaram que a radiação solar incidente e a velocidade do vento foram reduzidas pela cobertura antigranizo, em cerca de $30 \%$ e $40 \%$, respectivamente. Estas comparações entre condições microclimáticas, associadas à cobertura do pomar, podem explicar a maior similaridade de DPM entre diferentes estratos do dossel coberto, assim como a maior duração de molhamento no pomar coberto por tela, em relação ao dossel em céu aberto. As reduções de radiação solar e a velocidade do vento podem contrabalançar os efeitos da cobertura sobre a temperatura e a umidade relativa do ar. A redução da radiação tende a diminuir a temperatura e aumentar a umidade relativa do ar. Contudo, a redução da velocidade do vento dificulta a renovação do ar junto às superfícies, causando efeito contrário. A menor renovação do ar junto às superfícies do dossel poderia proporcionar maior DPM, com maior similaridade de valores entre os estratos do dossel coberto por tela.

É importante ressaltar que, na comparação entre ambientes, a duração do molhamento foliar diferiu nos estratos médio e superior dos dosséis, mas não diferindo no estrato inferior, apesar de o ambiente coberto ter apresentado maior média de DPM no dossel como um todo. Estas diferenças entre estratos justificam-se pela influência da velocidade do vento e da radiação solar, que tende a ser maior no topo das plantas, diminuindo em direção aos estratos inferiores do dossel. 
TABELA 1 - Duração média do período de molhamento (DPM), em h dia ${ }^{-1}$, em dosséis de macieiras 'Royal Gala' em céu aberto (D) e coberto por tela antigranizo (C). Vacaria-RS, 2009/2010.

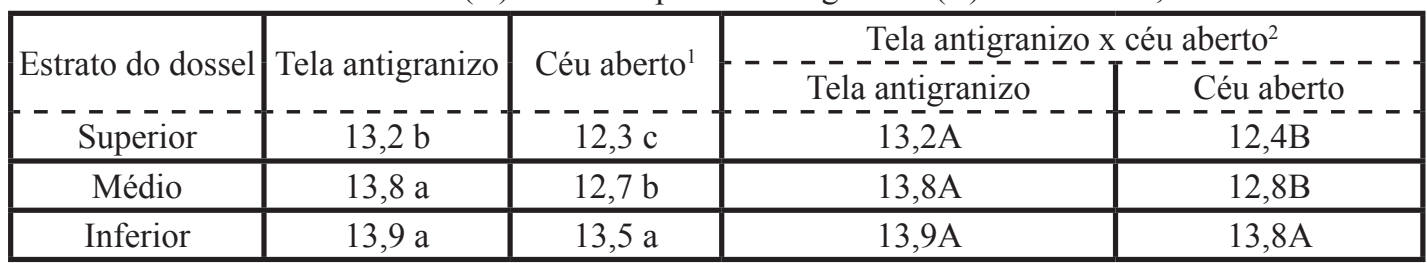

Médias seguidas na coluna pela mesma letra minúscula e na linha pela mesma letra maiúscula não diferem entre si, ao nível de 5\% de probabilidade de erro, pelo teste t para dados pareados; ${ }^{1}$ referente ao período mais longo de observação da DPM no ambiente em céu aberto (D); ${ }^{2}$ dados do período idêntico de observação da DPM, para cada estrato, nos dois ambientes coberto por tela antigranizo (C) e em céu aberto (D).

TABELA 2 - Diferenças médias de duração do molhamento foliar (DPM), em h dia-1, entre os estratos superior (S), médio (M) e inferior (I) de dosséis de macieiras 'Royal Gala', em céu aberto (descoberto) e sob tela antigranizo (coberto). Vacaria-RS, 2009/2010.

\begin{tabular}{lcccccc}
\hline & \multicolumn{3}{c}{ Descoberto } & \multicolumn{3}{c}{ Coberto } \\
\hline Diferenças Médias & I - M & I - S & M - S & I - M & I - S & M - S \\
\hline Todo Período & 0,8 & 1,2 & 0,4 & 0,2 & 0,7 & 0,7 \\
Dias com precipitação & 0,9 & 1,1 & 0,2 & 0,6 & 0,9 & 0,5 \\
Dias sem precipitação & 0,8 & 1,2 & 0,5 & $-0,1$ & 0,5 & 0,8 \\
\hline
\end{tabular}

TABELA 3 - Coeficientes de correlação (r) e índice de concordância de Willmott (D para duração do molhamento foliar (DPM) entre os estratos superior (S), médio (M) e inferior (I) de dosséis de macieiras 'Royal Gala', em céu aberto (descoberto) e sob tela antigranizo (coberto). Vacaria-RS, 2009/2010.

\begin{tabular}{lcccccc}
\hline \multirow{2}{*}{ Posição no dossel } & \multicolumn{2}{c}{ Todo o período } & \multicolumn{2}{c}{ Dias sem precipitação } & \multicolumn{2}{c}{ Dias com precipitação } \\
\cline { 2 - 7 } & $\mathrm{r}$ & $\mathrm{D}$ & $\mathrm{r}$ & $\mathrm{D}$ & $\mathrm{r}$ & $\mathrm{D}$ \\
\hline Descoberto & & & & & & \\
I e M & 0,91 & 0,94 & 0,90 & 0,94 & 0,92 & 0,99 \\
I e S & 0,89 & 0,92 & 0,89 & 0,92 & 0,88 & 0,92 \\
M e S & 0,90 & 0,96 & 0,89 & 0,96 & 0,89 & 0,95 \\
Coberto & & & & & & \\
I e M & 0,94 & 0,97 & 0,96 & 0,99 & 0,92 & 0,95 \\
I e S & 0,97 & 0,98 & 0,97 & 0,99 & 0,95 & 0,96 \\
M e S & 0,97 & 0,98 & 0,97 & 0,99 & 0,96 & 0,98 \\
\hline
\end{tabular}




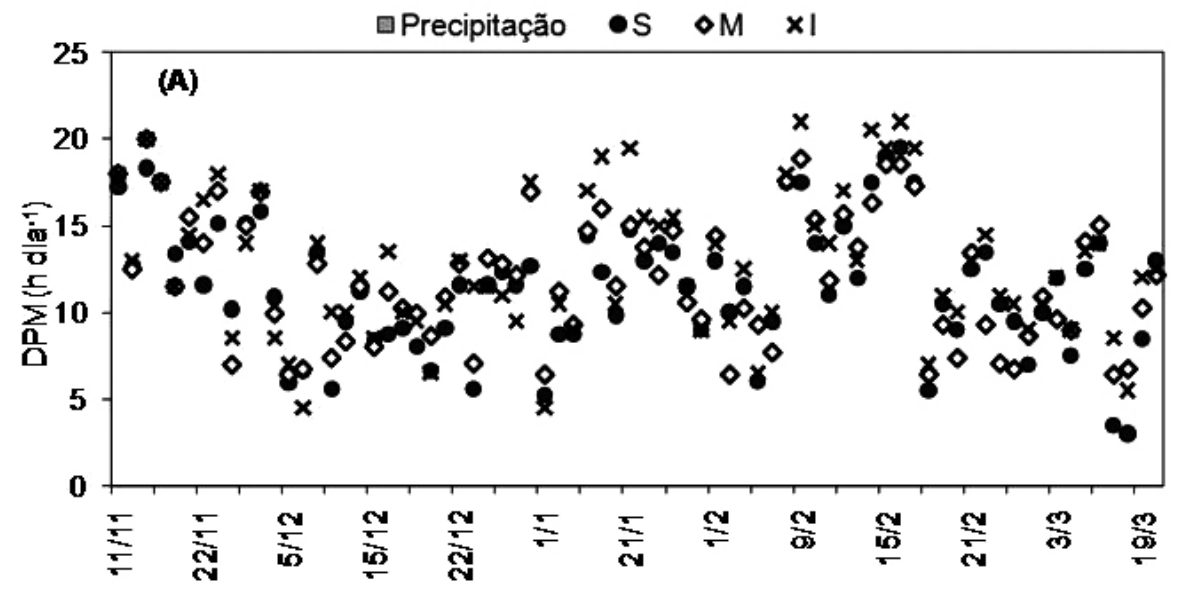

Dias semprecipitaçāo (2009/2010)

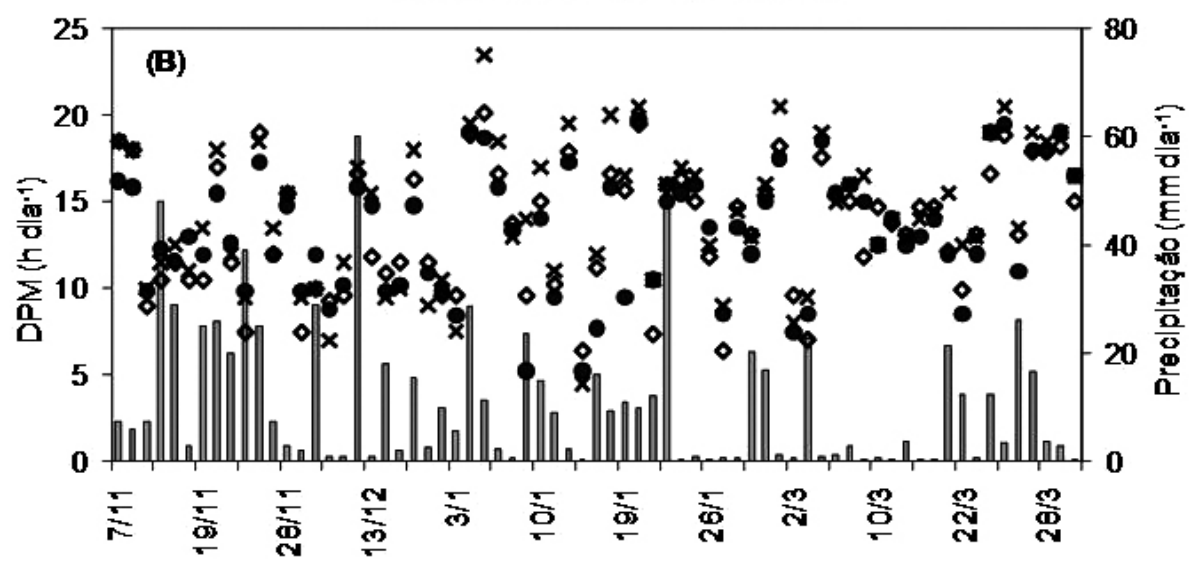

Dias comprecipilaçāo (2009/2010)

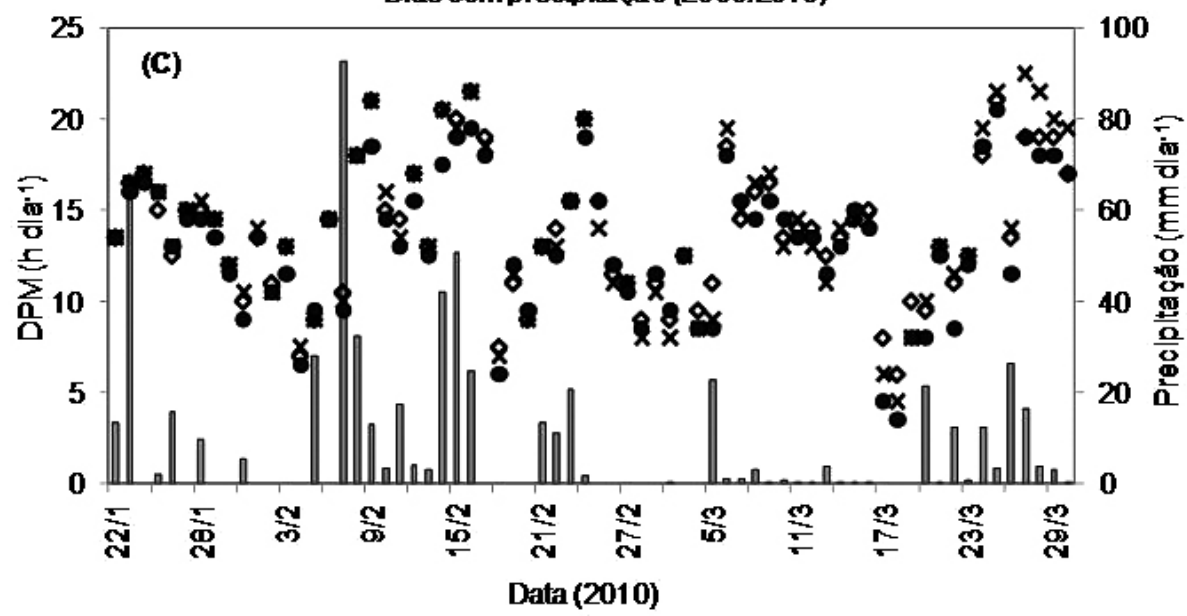

FIGURA 1 - Precipitação pluvial e duração do molhamento foliar (DPM) nos terços médio (M), inferior (I) e superior (S) de dosséis de macieiras 'Royal Gala', em céu aberto (A e B) e coberto por tela antigranizo (C). Vacaria-RS, 2009/2010. 

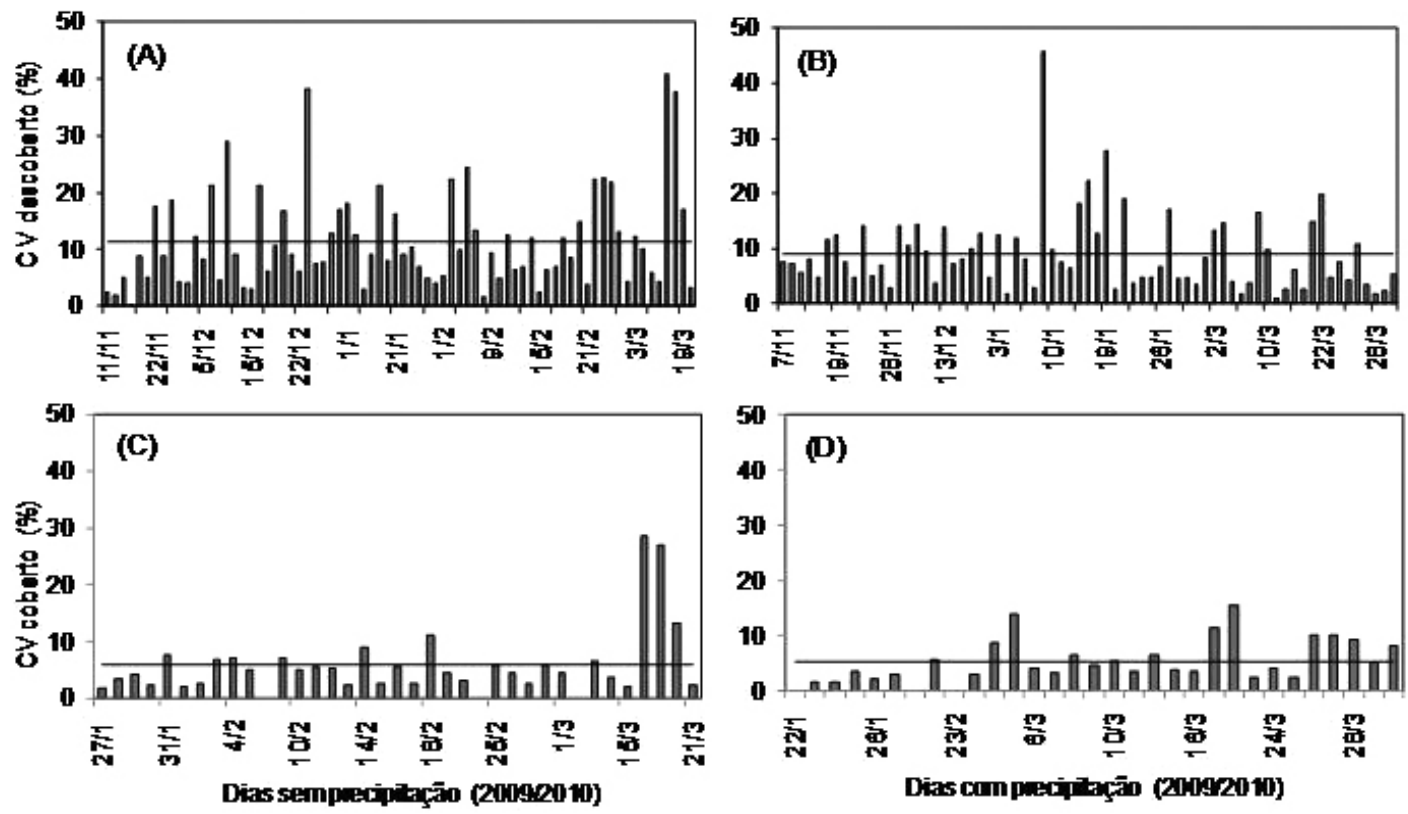

FIGURA 2 - Coeficientes de variação (CV) diário (barras) e médio (linha) para duração do molhamento foliar entre os terços médio, inferior e superior de dosséis de macieiras 'Royal Gala', em céu aberto (A e B) e coberto por tela antigranizo (C e D). Vacaria-RS, 2009/2010.

\section{CONCLUSÕES}

1-A duração do período de molhamento foliar (DPM) é maior em pomares de macieiras sob cobertura antigranizo que em céu aberto.

2-Em pomares de macieiras 'Royal Gala', utilizando-se de porta-enxerto de baixo vigor vegetativo, em alta densidade de plantas, conduzidas em linhas de cultivo espaçadas e abertas, a duração do molhamento foliar é maior no terço inferior do dossel, tanto em céu aberto como sob tela antigranizo.

3-Em pomares de macieira cobertos por tela antigranizo, as diferenças na duração do molhamento foliar entre estratos do dossel são menores que em pomares em céu aberto.

\section{REFERÊNCIAS}

AMARANTE, C. V. T.; STEFFENS, C. A.; MIQUELOTO, A.; ZANARDI, O. Z.; SANTOS, E. P. Disponibilidade de luz em macieiras'fuji' cobertas com telas antigranizo e seus efeitos sobre a fotossíntese, o rendimento e a qualidade dos frutos. Revista Brasileira de Fruticultura, Jaboticabal, v. 31, n. 3 , p. 664-670, 2009.

BATZER, J. C.; GLEASON, M. L.; TAYLOR, S. E.; KOEHLER, K. J.; MONTEIRO, J. E. B. A. Spatial heterogeneity of leaf wetness duration in apple trees and its influence on performance of a warning system for sooty blotch and flyspeck. Plant Disease, Saint Paul, v.92, n. 1, p. 164-170, 2008.

BERTON, O. Previsão e controle da sarna da macieria. In: REIS, E. M. Previsão de doenças de plantas. Passo Fundo: UPF, 2004. p. 291-316.

BOSCO, L. C.; CARDOSO, L. S.; PAULA, V. A.; BERGAMASCHI, H.; BRAUNER, P. C.; CASAMALLI, B.; SANTOS, V. R.; MARODIN, F. A.; SANTOS, H. P.; MARODIN, G. A. B. Microclima e qualidade de frutos de macieira sob tela antigranizo. In: REUNIÓN ARGENTINA Y VI LATINOAMERICANA DE AGROMETEOROLOGÍA, 13., 2010, Bahia Blanca. Anais... p. 43-44. 
CARDOSO, L. S.; BERGAMASCHI, H.; COMIRAN, F.; CHAVARRIA, G.; MARODIN, G. A. B.; DALMAGO, G. A.; SANTOS, H. P.; MANDELLI, F.. Padrões de interceptação de radiação solar em vinhedos com e sem cobertura plástica. Revista Brasileira de Fruticultura, Jaboticabal, v. 32, n. 1, p.161-171, 2010.

CARDOSO, L. S.; BOSCO, L. C.; PAULA, V. A.; BERGAMASCHI, H.; BRAUNER, P. C.; MARODIN, F. A.; SANTOS, V. R.; MARODIN, G. A. B. Alterações micrometeorológicas em pomares de macieira pelo uso de tela antigranizo. In: CONGRESSO BRASILEIRO DE AGROMETEOROLOGIA, 16., 2009, Belo Horizonte. Anais... 5 p.

CHAVARRIA, G.; SANTOS, H. P.; FELIPPETO, J.; MARODIN, G. A. B.; BERGAMASCHI, H. CARDOSO, L. S; FIALHO, F.B.. Relações hídricas e trocas gasosas em vinhedo sob cobertura plástica. Revista Brasileira de Fruticultura, Jaboticabal, v. 30, no.4, p.1022-1029, 2008.

CHAVARRIA, G.; SANTOS, H. P.; MANDELLI, F.; MARODIN, G. A. B.; BERGAMASCHI, H. CARDOSO, L. S. Caracterização fenológica e requerimento térmico da cultivar Moscato Giallo sob cobertura plástica. Revista Brasileira de Fruticultura, Jaboticabal, v. 31, no.1, p.119-126, 2009.

CHAVARRIA, G.; SANTOS, H. P.; SÔNEGO, O. R.; MARODIN, G. A. B.; BERGAMASCHI, H. CARDOSO, L. S.. Incidência de doenças e necessidade de controle em cultivo protegido de videira. Revista Brasileira de Fruticultura, Jaboticabal, v. 29, n.3, p.477-482, 2007.

DALLA MARTA, A.; ORLANDINI, S.; GHIRONI, M.; SABATINI, F. Influence of different sensor positions on leaf wetness measurements and their effect on the simulation of grapevine downy mildew (Plasmopara viticola). Időjárás, Budapeste, v. 108, n.4, p. 253-263, 2004.

HUBER, L.; GILLESPIE, T. J. Modeling leaf wetness in relation to plant disease epidemiology. Annual Review of Phytopathology, Palo Alto, v. 30, p. 553-577, 1992.

LEITE, G.B.; PETRI, J.L.; MONDARDO, M. Efeito da tela antigranizo em algumas características dos frutos de macieira. Revista Brasileira de Fruticultura, Jaboticabal, v. 24, n. 3, p. 714-716, 2002.
MacHARDY, W. E. Apple scab: biology, epidemiology and management. Saint Paul: The American Phytopathological Society, 1996. 545 p.

MacHARDY, W. E.; GADOURY, D. M. A revision of Mills' criteria for predicting apple scab infection periods. Phytopathology, Saint Paul, v. 79, n. 3, p. 304-310, 1989.

MADEIRA, A. C.; KIM, K. S.; TAYLOR, S. E.; GLEASON, M. L. A simple could-based energy balance model to estimate dew. Agricultural and Forest Meteorology, Amsterdam, v. 111, n.1, p. 55-63, 2002.

MILLS, W. D. Efficient use of sulphur dusts and sprays during rain to control apple scab. Ithaca: New York Agricultural Experimental Station, 1944. 4p. (Cornell Extension Bulletin, 630)

MONTEITH, J. L. Conservative behaviour in the response of crops to water and light. In: RABBINGE, R. (Ed.). Theoretical production ecology: reflections and prospects. Wageningen: Pudoc,1990. p. 3-16.

PEDROTTI, E. L.; VOLTOLINI, J. A. Enraizamento ex vitro e aclimatização do porta-enxerto de macieira M9. Revista Brasileira de Fruticultura, Jaboticabal, v. 23, n. 2, p. 234-239, 2001.

SENTELHAS, P. C. Duração do período de molhamento foliar: aspectos operacionais de sua medida, variabilidade especial em diferentes culturas e sua estimativa a partir do modelo de Penman-Monteith. 2004. 161 f. Tese (Livre-Docência)- Programa de Pós-Graduação em Agronomia, Escola Superior de Agricultura "Luiz de Queiroz", Universidade de São Paulo, Piracicaba, 2004.

SENTELHAS, P. C.; GILLESPIE, T. J.; BATZER, J. C.; GLEASON, M. L.; MONTEIRO, J. E. B. A; PEZZOPANE, J. R. M.; PEDRO JUNIOR, M. J. Spatial variability of leaf wetness duration in different crop canopies. International Journal of Biometeorology, New York, v. 49, n. 6, p. 363-370, 2005.

VALE, F. X. R; JESUS JUNIOR, W. C; ZAMBOLIM, L. Z. Epidemiologia aplicada ao manejo de doenças de plantas. Belo Horizonte: Perfil, 2004. $531 \mathrm{p}$.

WITTICH, K. P. Some remarks on dew duration on top of an orchard. Agricultural and Forest Meteorology, Amsterdam, v.72, n. 3-4, p.167-180, 1995. 